\title{
Incertidumbre de datos geoespaciales en la exploración petrolera
}

\section{Uncertainty of geospatial data in oil exploration}

\author{
Alexander Eduardo Inga Alva \\ Universidad Nacional Mayor de San Marcos, Facultad de Ingeniería de Sistemas e Informática. Lima, Perú. \\ Email alexander.inga@unmsm.edu.pe
}

\section{Resumen}

La presente investigación se ha enfocado en el análisis de la incertidumbre de datos geoespaciales en la etapa inicial de la exploración petrolera. A través de la evaluación de índices de confiablidad de distintos métodos de procesamiento de datos, se ha desarrollado un método que considera a la Información Geográfica Voluntaria (IGV) como una nueva fuente de datos y a la Geodatabase (GDB) como el repositorio donde se ha validado dicha información. Con la creación del artefacto "Uncertainty Sphere" que se ha basado en los algoritmos de la Guía para la Expresión de la Incertidumbre y Medición (GUM) y las recomendaciones de ISO 19157:2013, Calidad de Datos Geográficos, se ha delimitado el Espacio de la Incertidumbre de los datos recolectados. La aplicación de un Sistema de Información Geográfica (SIG) ha gestionado los datos de entrada (IGV), el procesamiento geoespacial (artefactos), el almacenamiento (GDB) y los productos informativos (mapas). Como caso de estudio, se ha evaluado la incertidumbre del geoposicionamiento del Pozo Capahuari Sur 3 de la empresa Frontera Energy y se han validado seis posibles ubicaciones usando GPSLogger y once con GPSMap60CSx. Estos resultados han permitido a la empresa considerar mejores escenarios para la perforación de futuros pozos exploratorios.

Palabras clave: Incertidumbre; Colaboración Abierta Distribuida; Sistema de Información Geográfica (SIG).

\begin{abstract}
The present investigation has focused on the analysis of the uncertainty of geospatial data in the initial stage of oil exploration. Through the evaluation of reliability indices of different data processing methods, a method has been developed that considers Voluntary Geographical Information (VGI) as a new data source and Geodatabase (GDB) as the repository where this information has been validated. With the creation of "Uncertainty Sphere" artifact that has been based on the algorithms of Guide for the Expression of Uncertainty and Measurement (GUM) and the recommendations of ISO 19157: 2013, Geographic Data Quality, Uncertainty Space has been delimited from data collected. The application of a Geographic Information System (GIS) has managed input data (IGV), geospatial processing (artifacts), storage (GDB) and information products (maps). As a case of study, geopositioning uncertainty of Capahuari Sur 3 well of Frontera Energy company has been evaluated and six locations have been validated with GPSLogger and eleven with GPSMap60CSx. These results have allowed the company to consider better scenarios for the drilling of future exploratory wells.
\end{abstract}

Keywords: Uncertainty; Crowdsourcing; Geographic Information System (GIS).

Inga A. Incertidumbre de Datos Geoespaciales en la Exploración Petrolera. Revista Peruana de Computación y Sistemas 2018 1(2):41-54. http://dx.doi. org/10.15381/rpcs.v1i2.15378

(c) Los autores. Este artículo es publicado por la Revista Peruana de Computación y Sistemas de la Facultad de Ingeniería de Sistemas e Informáticade la Universidad Nacional Mayor de San Marcos. Este es un artículo de acceso abierto, distribuido bajo los términos de la licencia Creative Commons Atribucion - No Comercia_Compartir Igual 4.0 Internacional. (http://creativecommons.org/licenses/by-nc-sa/4.0/) que permite el uso no comercial, distribución y reproducción en cualquier medio, siempre que la obra original sea debidamente citada. 


\section{Introdución}

Desde tiempos inmemoriales el petróleo ha sido conocido y usado por los primeros hombres: "Y el valle de Sidim estaba lleno de pozos de asfalto." (Génesis 14:10). En el imperio Incaico, se le conoció como "Copé" y se usaba como impermeabilizante de cántaros y vasijas de barro y, posteriormente, de las embarcaciones españolas. A nivel mundial, fue el coronel William Drake quien perforó el primer pozo con fines comerciales en 1859 en la localidad de Oil Creek, Titusville en el estado norteamericano de Pensylvania. En 1863, en el norte del Perú, en la zona llamada "La Brea" se perforó el primer pozo sudamericano [1]. A partir de entonces, una complicada actividad petrolera se ha desarrollado en nuestro país, como se registra en el año 1911 cuando la empresa London \& Pacific Petroleum Co. por una mala praxis en sus valoraciones fue denunciada por malversación. Después de cuatro años, el gobierno peruano exigió reevaluar su propiedad y, aunque en 1921 se sometió a arbitraje por exigencias del Gobierno Británico, no se consideró "la tenencia del subsuelo". En 1924, la Internacional Petroleum Company (IPC) adquirió los derechos sobre "La Brea" y casi por medio siglo los conflictos sobre el "Laudo de la Brea y Pariñas" se mantuvieron. En 1968, tras establecerse un mutuo acuerdo entre el Estado y la compañía privada, el negociador estatal denunció posteriormente la desaparición de la famosa "página 11" del acuerdo, lo que fue tomado como pretexto por el General Juan Velasco Alvarado para derrocar al gobierno constitucional del arquitecto Fernando Belaunde Terry, expropiando IPC y constituyendo a PETROPERÚ. Años más adelante, IPC fue compensado por el mismo gobierno militar que también recurriría, a pesar de su nacionalismo, a tecnologías y empresas extranjeras para la exploración petrolera en la selva amazónica a través de la creación del Modelo Peruano. Este modelo atrajo a dieciocho empresas que suscribieron contratos exploratorios, pero fue sólo la empresa Occidental Petroleum la que hizo un descubrimiento (en el Lote 1-A y 1-B) [2]. Con el tiempo, estos lotes se fusionaron formando el 1-AB que en 1985 pasó a la empresa Pluspetrol por 30 años (Perupetro, 2012 Memoria Anual). Hoy en día, ese lote ha sido renombrado como el Lote 192 siendo operado por la empresa Frontera Energy.

Con respecto a la disponibilidad de la información, a nivel internacional se ha manejado altos niveles de acceso a bases de datos de diferentes instituciones estatales. Sin embargo, la calidad que estas han presentado son uno de los grandes problemas, tal como lo indica el proyecto CYTED-IDEDES 606PI0294 sobre la "Evaluación y Potenciación de Infraestructuras de Datos Espaciales (IDE) para el desarrollo sostenible en América Latina y el Caribe" [3] donde se ha señalado que: "sería interesante evaluar en futuros estudios la calidad de la información, más allá de su propia disponibilidad". A nivel nacional, según Resolución Ministerial 325-2007PCM (Presidencia del Consejo de Ministros) se creó la Infraestructura de Datos Espaciales del Perú (IDEP) que es la suma de directrices, modelos, medios y técnicas es- paciales que permiten el uso de información geoespacial del país con el propósito de generar bienestar, sostenibilidad y soporte gerencial. El sustento de esta arquitectura proviene de entidades públicas y privadas productoras de información geográfica, que brindan esta información vía internet con un mínimo de protocolos y especificaciones normalizadas (GEOIDEP, 2018). A pesar de esta iniciativa por parte del Estado para integrar a todos sus productores de datos geográficos, aún se tiene que la información, procedente de los bancos de datos espaciales, de distintas fuentes públicas por ser antiguas, en muchos casos, están mal georreferenciadas, no presentan georreferencia, carecen de reproyección o fueron creadas por terceros en sistemas propietarios. A esto se suma la falta de articulación institucional, la duplicidad de datos y la definición de las competencias institucionales (GEOIDEP, 2017) [4]. Considérese el siguiente ejemplo: ¿cuántos centros poblados existen actualmente en el Perú? Distintas instituciones estatales responden de forma diferente (Ver Tabla 1 ).

Tabla 1. Bases de datos de centros poblados

\begin{tabular}{lc} 
Institución & Centros Poblados \\
INEI* & 98011 \\
MINEDU** & 116495 \\
ONPE*** & 69951 \\
MINEM**** & 67508 \\
Regiones & Datos Propios \\
\hline
\end{tabular}

*Instituto Nacional de Estadística e Informática, **Ministerio de Educación, ${ }^{* * *}$ Oficina Nacional de Procesos Electorales, ${ }^{* * * *}$ Ministerio de Energía y Minas

Asimismo, datos más modernos adolecen de un control de calidad que valide su correcto posicionamiento y que se ajuste a las normativas actuales de la Red Geodésica Nacional, que según RJ No 086-2011-IGN/ OAJ/DGC, actualiza el marco de referencia de ITRF 1994 (época 1995-4) al ITRF 2000 (WGS84). De persistir esta problemática, la incertidumbre crecerá (entropía) al querer, por ejemplo, contrastar datos actuales con datos históricos no encontrándose un referente espacial confiable.

A pesar de que existe en la industria del petróleo documentación y mayor conciencia del uso de métodos probabilísticos, ¿por qué no ha habido una adopción más extendida?, ¿por qué la industria aparentemente todavía subestima la incertidumbre en sus distintas etapas? Parte de la respuesta a estas preguntas es que algunas empresas que han intentado cuantificar la incertidumbre y el análisis de decisión han experimentado la "Confusión inducida por la incertidumbre" como resultado de una aplicación incorrecta [5]. Otro factor de mayor relevancia ha sido: la falta de pruebas claras de que el incremento de la cuantificación de la incertidumbre y del análisis de decisiones, haya conducido a una mejora significativa en la toma de decisiones y en el rendimiento en la industria. Cabe resaltar, que la mayoría de los beneficios que se citan en los procesos de cuantificación 
realmente son de naturaleza cualitativa, motivo por el cual muchas empresas son reacias a adoptar estos procesos [6].

El objetivo principal del presente estudio ha sido establecer un modelo para la gestión de la incertidumbre geoespacial en la etapa inicial de exploración (estudios geológicos). La incertidumbre se refiere al rango de probabilidades en el que algunas condiciones pueden existir u ocurrir [7]. El análisis se centra en medir la incertidumbre de las fuentes de datos en sus componentes asociados a: precisión de coordenadas proyectadas, georreferenciación y precisión de elevación. La evaluación se ha basado en la asignación de índices de confiabilidad adoptadas según la precisión del dispositivo, equipamiento o procedimiento con que se han realizado las mediciones. De esta manera, es gestionada la confiabilidad de la ubicación de los pozos petroleros exploratorios, generando a la empresa ventaja competitiva y disminución de riesgos.

\section{Marco Teórico}

\subsection{Modelo Exploratorio}

A nivel internacional se han desarrollado en las últimas décadas normas que direccionan los contratos entre el ente estatal y las compañías de hidrocarburos que buscan explorar y explotar estos recursos. De esta forma, tanto los contratos de: asociación, servicios, operación, licencia o estrategia, enfrentan las siguientes etapas:

2.1.1 Riesgos Asociados: en cada modelo que se pretenda utilizar, los riesgos propios de la goelogía, los riesgos financieros, los riesgos sociales y los riesgos operativos en las actividades de exploración, corren por cuenta del inversor privado.

2.1.2 Periodos Sucesivos: el proceso exploratorio está dividido en períodos sucesivos y que son otorgados al inversionista para que este desarrolle un compromiso gradual de nuevos trabajos exploratorios. De esta manera, conforme va obteniendo mayor información, el inversionista puede continuar con la exploración o eventualmente, abandonar el lote.

2.1.3 Descubrimiento Comercial: con una exploración exitosa, el inversionista está en la capacidad de declarar que existen las reservas mínimas requeridas para su comercialización, lo que deber estar acorde al justiprecio del mercado petrolero.

2.1.4 Programas Obligatorios: hace referencia a los trabajos básicos o elementales que el inversionista está obligado a cumplir. Para ello, el Estado solicita garantías económicas a través de la banca, que son ejecutadas en caso de no cumplir con la tarea acordada. Por ejemplo: Programa de levantamiento de "X" Km de Sísmica 2D.

2.1.5 Tipos de Retribuciones: en el Perú existen dos sistemas: retribución en dinero y retribución en especies (gas natural o petróleo crudo).

\subsection{Sistemas de Información Geográfica (SIG)}

En la década del sesenta, el Dr. Roger Tomlinson, conocido como el Padre del SIG implementó el primer Sistema de Información Geográfica de Canadá (CGIS).

- Según ESRI (Environmental Systems Research Institute), "es un sistema para la gestión, análisis y visualización de conocimiento geográfico que se estructura en diferentes conjuntos de información" [8].

- Burrough, (1986): "Un potente conjunto de herramientas para recolectar, almacenar, recuperar a voluntad, transformar y presentar datos espaciales procedentes del mundo real" [9].

- Una definición más aceptada es la dada por (NCGIA, National Center for Geographic Information and Analysis, 1989), "Sistema de hardware, software y procedimientos diseñado para realizar la captura, almacenamiento, manipulación, análisis, modelización y presentación de datos referenciados espacialmente para la resolución de problemas complejos de planificación y gestión" [10].

\subsection{Información Geográfica Voluntaria (IGV)}

El concepto de Información Geográfica Voluntaria (IGV) fue acuñado por Michael F. Goodchild en el año 2007 y es la información geográfica creada o recogida por voluntarios de forma organizada, que no son necesariamente especialistas y publicada en línea [11].

Según Haklay (2013) [12], el concepto general de "crowdsourcing" es clasificado en cuatro niveles:

- Nivel I: Población

- Ciudadanos como sensores

- Computación voluntaria distribuida

- Nivel II: Inteligencia distribuida

- Ciudadanos como intérpretes básicos

- Pensamiento voluntario

- Nivel III: Ciencia participativa

- Participación en la definición del problema y la recolección de datos

- Nivel IV: Ciencia ciudadana extrema

- Ciencia colaborativa - define problema, recolecta datos y Análisis.

\subsection{Incertidumbre}

El término "incertidumbre" ha sido asociado a la indecisión y, más aún, se le ha relacionado a la confiabilidad de la medición de un objeto o proceso, tratándose de medir la utilidad de su resultado, usando por ejem- 
plo, la desviación típica, en casos particulares (GUM 2.2.1). La Guía para la expresión de la incertidumbre de la medida (GUM) ha permitido agrupar los elementos de la incertidumbre de dos maneras: método de evaluación tipo A y método de evaluación tipo B, las cuales se diferencian en su forma de evaluar las componentes de incertidumbre, basándose ambas en distribuciones de probabilidad. (GUM 3.3.3) [13].

2.4.1. Incertidumbre de Tipo $A(U a)$ : este método está basado en el estudio de la estadística generada por un conjunto de observaciones. (GUM 2.3.2). De esta forma, la mejor valoración esperada " $\mu \mathrm{q}$ " de una cantidad "q", con "n" mediciones autónomas "qk" es la media aritmética $\bar{q}$ (GUM 4.2.1):

$$
\bar{q}=\frac{1}{n} \sum_{k=1}^{n} q k
$$

Por otro lado, la varianza " $\sigma^{2 \text { " }}$ de esta distribución $\bar{q}$ viene dada por (GUM 4.2.2):

$$
s^{2}\left(q_{k}\right)=\frac{1}{n-1} \sum_{k=1}^{n}(q k-\bar{q})^{2}
$$

Tanto la desviación estándar como la varianza nos indican como se dispersan los valores alrededor de la media $\bar{q}$ y pueden ser usadas para medir la incertidumbre (GUM 4.2.3):

$$
s^{2}(\bar{q})=\frac{s 2(q k)}{n}
$$

2.4.2. Incertidumbre de Tipo $B(U b)$ : en este caso la estimación de las magnitudes no proceden de observaciones repetidas. $\mathrm{La}$ incertidumbre está basada en la información de variación de Xi. La información puede provenir de:

- Mediciones pasadas (históricos).

- Conocimiento de la conducta y atributos de componentes y herramientas.

- Especificaciones técnicas del producto.

- Certificados referentes a la graduación.

- Información relacionada a la certidumbre de las medidas encontradas en handbooks (GUM 4.3.1).

2.4.3. Incertidumbre Combinada $\left(U_{c}\right)$ : es el resultado de la evaluación de la incertidumbre A y la incertidumbre B, a través de la siguiente fórmula simplificada (GUM 5.1.2):

$$
U c=\sqrt{U a^{2}+U b^{2}}
$$

2.4.4. Incertidumbre Expandida (U): es el intervalo de confianza alrededor de una medición y que genera una cierta cantidad de mesurandos que puede satisfacer el valor de la medida. La Incertidumbre Expandida (U) se obtiene a través de la siguiente fórmula GUM 6.2.1):

$$
U=k u c
$$

dónde: "k" es un factor de cobertura.

Ahora bien, en el caso de una variable aleatoria unidimensional $\mathrm{Z}$ puede expandirse a tres dimensiones siempre y cuando se obtengan tres valores para la magnitud medida. El resultado está dado por la tupla X, Y, $Z$. La equivalencia con el intervalo de confianza en una dimensión es el volumen de confianza, que usualmente se describe como una esfera en torno a la mejor estimación del valor verdadero. La probabilidad de que el valor verdadero esté situado dentro de este volumen se calcula mediante la integración de volúmenes sobre la función de densidad tridimensional de la distribución normal. Un volumen esférico es caracterizado por su radio. Este radio es usado como medida de la exactitud de variables aleatorias tridimensionales (ISO 19157:2013, G.3.4) [14].

\section{Estado del arte (resumen)}

La revisión de distintas metodologías ha permitido evaluar la confiabilidad de los datos de acuerdo a juicio de expertos y se ha ponderado según la escala de Likert (ver Tabla 2) de la siguiente manera: Métodos de Incertidumbre de Datos [15] [16] [17] [18] (ver Tabla 4), Métodos de Transformación de Coordenadas [19] [20] [21] [22] (ver Tabla 5), Métodos de Geodatabase [23] [24] [25] (ver Tabla 6), Métodos de Calidad de Datos [26] [27] (ver Tabla 7) y Métodos de Afines [28] [29] [30] (ver Tabla 8).

Tabla 2. Escala de Likert para comparación de métodos

\begin{tabular}{cc} 
& Escala Likert \\
1 & Malo \\
2 & Regular \\
3 & Normal \\
4 & Bueno \\
5 & Excelente \\
\hline
\end{tabular}

El estudio de las matrices que ha sido direccionado por el método Best-Worst-Method (BWM) o Método del Mejor-Peor [31], ha permitido concluir que: el Método de Geodatabase (Control de calidad) obtiene el mayor puntaje (13 puntos) según juicio de expertos, identificándolo claramente como el método apropiado para gestionar la incertidumbre de los datos geográficos. Caso contrario ocurre con la elección del Método de Crowdsourcing, que a pesar de haber obtenido un puntaje bajo ( 6 puntos) dentro de la categoría de métodos afines, ha sido seleccionado para evidenciar que es susceptible a mejoras si es considerado como una nueva fuente de datos en el proceso de recolección en campo, como también ha sido precisado por Saberi, B. y Ghadiri, N. [27]. 


\section{1. Índices de Confiabilidad}

En esta sección se ha descrito los índices de confiabilidad de las fuentes elegidas basados en la precisión del dispositivo, equipamiento o procedimiento, con que se realizan las mediciones (ver Tabla 3).

Tabla 3. Índices de confiabilidad

\begin{tabular}{|c|c|c|}
\hline $\begin{array}{l}\text { Fuente de } \\
\text { Incertidumbre }\end{array}$ & Punto & Descripción de índices \\
\hline \multirow{5}{*}{$\begin{array}{l}\text { Precisión de } \\
\text { Coordenadas } \\
\text { Proyectadas (XY) }\end{array}$} & 1 & Precisión desconocida \\
\hline & 2 & > 50 m (Mapa topográfico) \\
\hline & 3 & 10-50 m (GPS Asistido-AGPS) \\
\hline & 4 & 1-10 m (GPS Navegador-GPS) \\
\hline & 5 & 0-1 m (GPS Diferencial-DGPS) \\
\hline \multirow{5}{*}{ Geo referencia } & 1 & Sin georreferenciación (solo visual) \\
\hline & 2 & Georreferenciación Falso positivo. \\
\hline & 3 & Georreferenciación (Benchmark) \\
\hline & 4 & Georreferenciación (Est. Pasiva) \\
\hline & 5 & Georreferenciación (Est. Permanente) \\
\hline \multirow{5}{*}{$\begin{array}{l}\text { Precisión de } \\
\text { Elevación (Z) }\end{array}$} & 1 & Precisión desconocida \\
\hline & 2 & > 50 m (Modelo Elevación Digital) \\
\hline & 3 & 10-50 m (GPS Asistido-AGPS) \\
\hline & 4 & 1-10 m (GPS Navegador-GPS) \\
\hline & 5 & 0-1 m (GPS Diferencial-DGPS) \\
\hline
\end{tabular}

\section{Metodología}

Se ha utilizado un Sistema de Información Geográfica (SIG) para gestionar: los procesos de entrada de datos tradicionales y no tradicionales (crowdsourcing), el geoprocesamiento de datos a través de índices de confiabilidad y uso de artefactos para tratar la incertidumbre (donde se ha usado las directrices de la Guía para la expresión de la Incertidumbre GUM-2008 y las recomendaciones del ISO 19157:2013), almacenamiento de la información validada en la Geodatabase y la creación de productos informativos (mapas).

\subsection{Visión General del Método Propuesto}

En esta sección se muestra de forma general el método propuesto sin profundizar en los detalles, señalando las partes que lo componen y de qué forma se insertan en las etapas del modelo utilizado (ver Figura 1).

\subsubsection{Fuente de Datos}

Básicamente son dos las principales:

Data Geoespacial Tradicional

Tanto la data pública y privada, en formato de data vectorial se ha trabajado en:

- Shapefiles (shp) y Features

- Archivos CAD (dwg, dxf)

\section{- $\quad$ GPS y DGPS}

y la data tabular/atributiva en:

- Tablas (xlsx, dbf, txt)

Data Geoespacial No Tradicional

Es data producto de la colaboración participativa voluntaria. Los datos en formato "gpx" recolectados con:

- Unidades GPS

- Celulares inteligentes con la aplicación GPSLogger

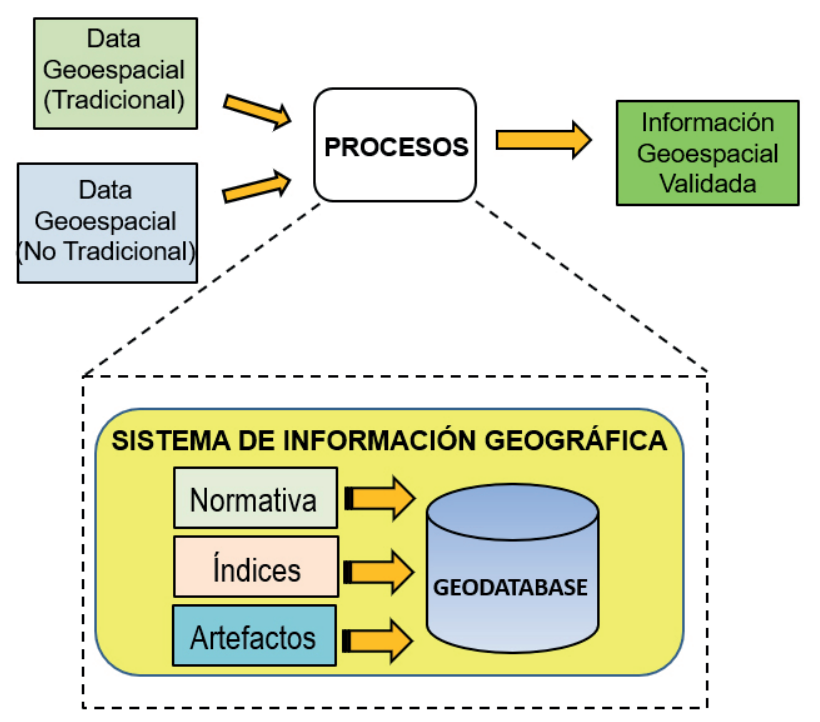

Fig. 1. Visión General del Método Propuesto

\subsubsection{Geoprocesos}

Estos procesos geoespaciales se hallan enmarcados dentro de las normativas del GUM 2008 e ISO 19157:2013 lo que ha permitido la asignación de los índices de confiablidad para evaluar las tres fuentes de incertidumbre elegidas:

- Precisión de la Coordenadas Proyectadas

- Georreferenciación en el mapa

- Precisión de la Elevación (Z).

Paralelamente se ha desarrollado los artefactos: "GPXToFC", "FC2D to FCMean3D" y "Uncertainty Sphere".

\subsubsection{Los Productos Informativos}

Una vez que la información geoespacial ha sido validada, se obtiene una información con incertidumbre controlada, lo que ha permito manejar el riesgo y catalogarla como apta para la toma de decisiones directivas. Este resultado está representado en productos informativos digitales (mapas) que son almacenados en una Geodatabase corporativa [32]. 
Tabla 4. Métodos de Incertidumbre de Datos

\begin{tabular}{lcccc} 
FUENTE DE INCERTIDUMBRE & MÉTODO INCERTIDUMBRE DE DATOS & $\begin{array}{c}\text { Mes } \\
\text { Representación }\end{array}$ & $\begin{array}{c}\text { Taxonomías prácticas } \\
\text { para datos SIG }\end{array}$ \\
Precisión de coordenadas proyectadas & 2 & 2 & 4 & 2 \\
Georreferenciación & 2 & 2 & 2 & 2 \\
Precisión de la Elevación $(\mathrm{Z})$ & 2 & 2 & 2 & 2 \\
PUNTAJE & 6 & 6 & 8 & 6 \\
\hline
\end{tabular}

Tabla 5. Métodos Transformación de Coordenadas

\begin{tabular}{lcccc} 
FUENTE DE INCERTIDUMBRE & \multicolumn{2}{c}{$\begin{array}{c}\text { MÉTODO TRANSFORMACIÓN DE COORDENADAS } \\
\text { Geo }\end{array}$} & $\begin{array}{c}\text { Algoritmo Búsqueda } \\
\text { Diferencial }\end{array}$ & $\begin{array}{c}\text { Clúster tramas } \\
\text { Programación Genética }\end{array}$ \\
Precisión de coordenadas proyectadas & 5 & 2 & 2 & 5 \\
Georreferenciación & 2 & 5 & 5 & 2 \\
Precisión de la Elevación (Z) & 5 & 2 & 2 & 2 \\
PUNTAJE & $\mathbf{1 2}$ & $\mathbf{9}$ & $\mathbf{9}$ & 9 \\
\hline
\end{tabular}

Tabla 6. Métodos de Geodatabase

\begin{tabular}{lccc} 
FUENTE DE INCERTIDUMBRE & & MÉTODO GEODATABASE \\
Precisión de coordenadas proyectadas & DB4GeO & Desnormalización & Control de Calidad GDB \\
Georreferenciación & 4 & 3 & 4 \\
Precisión de la Elevación (Z) & 3 & 3 & 5 \\
PUNTAJE & 3 & 10 & $\mathbf{1 3}$ \\
\hline
\end{tabular}

Tabla 7. Métodos de Calidad de Datos

\begin{tabular}{|c|c|c|}
\hline \multirow[b]{2}{*}{ FUENTE DE INCERTIDUMBRE } & \multicolumn{2}{|c|}{ MÉTODO CALIDAD DE DATOS } \\
\hline & Limpieza de datos estadístico & $\begin{array}{c}\text { Calidad de datos espaciales } \\
\text { Basado en muestra }\end{array}$ \\
\hline Precisión de coordenadas proyectadas & 4 & 3 \\
\hline Georreferenciación & 2 & 2 \\
\hline Precisión de la Elevación (Z) & 2 & 3 \\
\hline PUNTAJE & 8 & 8 \\
\hline
\end{tabular}

Tabla 8. Métodos Afines

\begin{tabular}{lccc} 
FUENTE DE INCERTIDUMBRE & \multicolumn{1}{c}{ MÉTODOS AFINES } \\
& KNIME & Crowdsourcing Spatial & Análisis Topológico \\
\hline Precisión de coordenadas proyectadas & 2 & 2 & 4 \\
Georreferenciación & 2 & 2 & 3 \\
Precisión de la Elevación (Z) & 2 & 2 & 2 \\
PUNTAJE & 6 & 6 & 9 \\
\hline
\end{tabular}

\subsection{Visión Específica del Método Propuesto}

En esta sección se muestra de forma específica el método propuesto, profundizando sobre todo en los elementos del geoprocesamiento, tales como: precisión del equipo, pesos asignados y artefactos (ver Figura 2).

\subsubsection{Precisión de equipo y asignación de pesos}

Para la asignación de pesos a los índices de confiabilidad, se ha determinado utilizar la precisión de los equipos usados en la medición de las coordenadas [33].
Basado en la fórmula de la media aritmética ponderada, tenemos:

$$
\begin{aligned}
& \mathrm{W}=(\mathrm{I} 1+\mathrm{I} 2+\mathrm{I} 3) /(\mathrm{I} 1 \times \mathrm{P}+\mathrm{I} 2 \times \mathrm{P}+\mathrm{I} 3 \times \mathrm{P}) \\
& \text { dónde: }
\end{aligned}
$$

I1 = Índice de Confiablidad de la Precisión de la Coordenadas Proyectadas.

I2 = Índice de Confiablidad de la Georreferenciación.

I3 = Índice de Confiablidad de la Precisión de la Elevación.

$\mathrm{P}=$ Precisión del equipo en el momento del registro.

$\mathrm{W}=$ Peso 


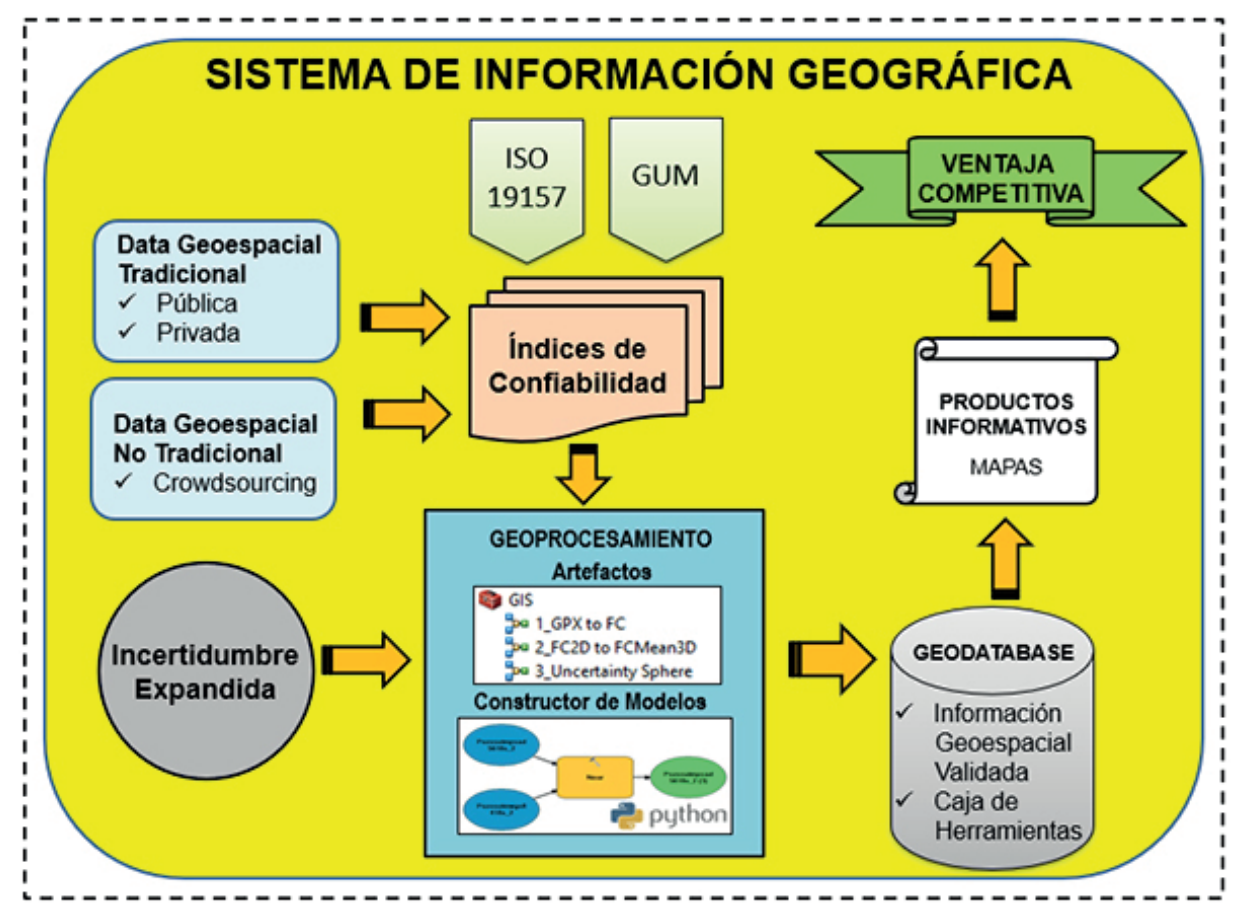

Fig. 2. Visión Específica del Método Propuesto

\subsubsection{Evaluación de las incertidumbres de medición}

Para el caso de una esfera se tiene que su volumen está caracterizado por su radio, el cual se usa como medida de la exactitud de variables aleatorias tridimensionales. Asimismo, la Guía para la expresión de la Incertidumbre de la medida (GUM) ha permitido analizar los elementos de las incertidumbre a través de la Incertidumbre Tipo A, B, Combinada y Expandida. El resultado de esta medición se expresa de la siguiente manera:

$$
\mathrm{Y}=\mathrm{y} \pm \mathrm{u}
$$

Esto significa que el resultado de la medición ha sido parametrado en un intervalo que varía entre -u y $+\mathrm{u}$, albergando las posibles soluciones del mesurando "Y". Además, se debe indicar explícitamente las unidades de "y".

\subsubsection{Artefactos}

En esta sección se ha descrito tres artefactos que están basados en el lenguaje de programación Python 2.7 [34] y están almacenados en la caja de herramientas GIS. Estos artefactos son: "GpxToFC", "FC2D to FCMean3D” y "Uncertainty Sphere” (ver Figura 3).

\section{GIS}

\section{1_GPX to FC 2_FC2D to FCMean3D 3_Uncertainty Sphere}

Fig. 3. Artefactos creados en ArcGIS.

GpxToFC: este artefacto ha permitido dos geoprocesos: la conversión de los puntos GPS recolectados por la aplicación GPSLogger desde su formato nativo "gpx" al formato vectorial "shp" (shapefile) y la reproyección de coordenadas del Sistema Geográfico WGS84 al Sistema UTM WGS84 - 18S. Los geoprocesos se han detallado en el constructor de modelos (ver Figura 4 ).

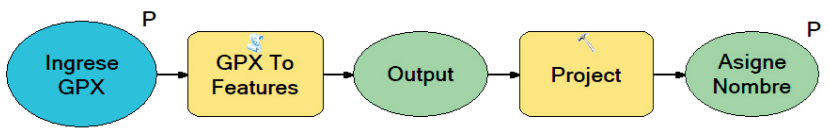

Fig. 4. Constructor de modelos de GpxToFC

FC2DtoFCMean3D: este artefacto ha permitido tres geoprocesos: el cálculo de las coordenadas de los elementos tipo puntos (en este caso los pozos exploratorios), la conversión de los puntos bidimensionales a tridimensionales y el cálculo de la coordenada del centro medio ponderado donde se ha considerado el "peso" calculado a partir de los índices de confiabilidad. Los geoprocesos se han detallado en el constructor de modelos (ver Figura 5 ).

Uncertainty Sphere: este artefacto ha permitido tres geoprocesos: crea la Esfera de Incertidumbre (elemento de tipo MultiPatch) a partir de la Incertidumbre Expandida, selecciona los pozos ubicados dentro de la referida esfera y se exporta a una nueva capa vectorial denominada "Pozos Validados". Los geoprocesos se han detallado en el constructor de modelos (ver Figura 6 ).

Finalmente, la información validada es almacenada en la Geodatabase empresarial (ver Figura 7).

$$
\begin{aligned}
& \boxminus \square \text { GEODATABASE.gdb } \\
& \boxminus \text { 它 FDS } \\
& \vdots \text { _CoordenadasValidas }
\end{aligned}
$$

Fig. 7. Coordenadas válidas almacenadas en Geodatabase 


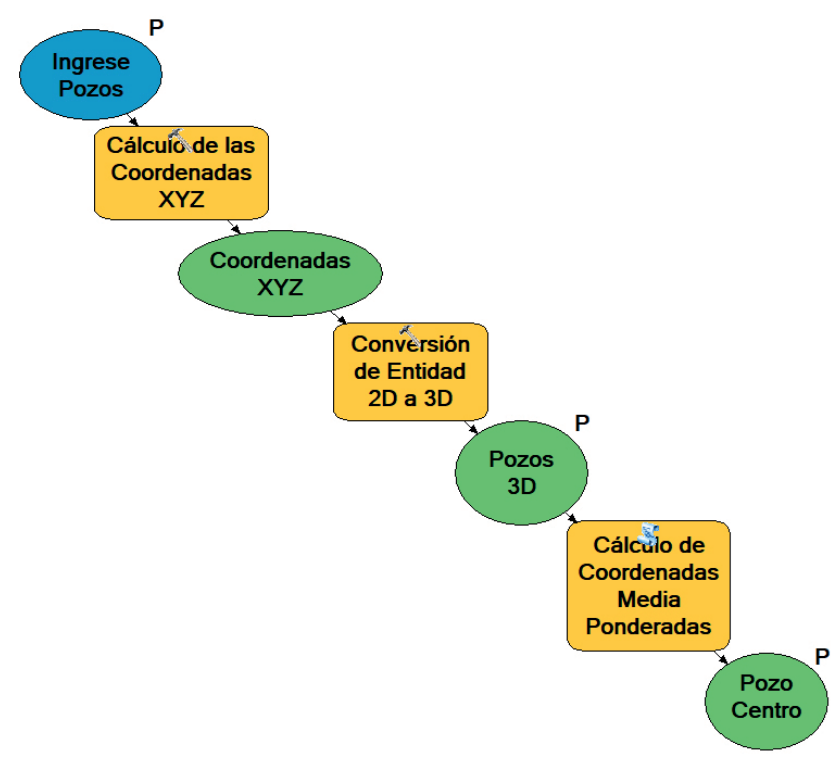

Fig. 5. Constructor de modelos de FC2DtoMean3D

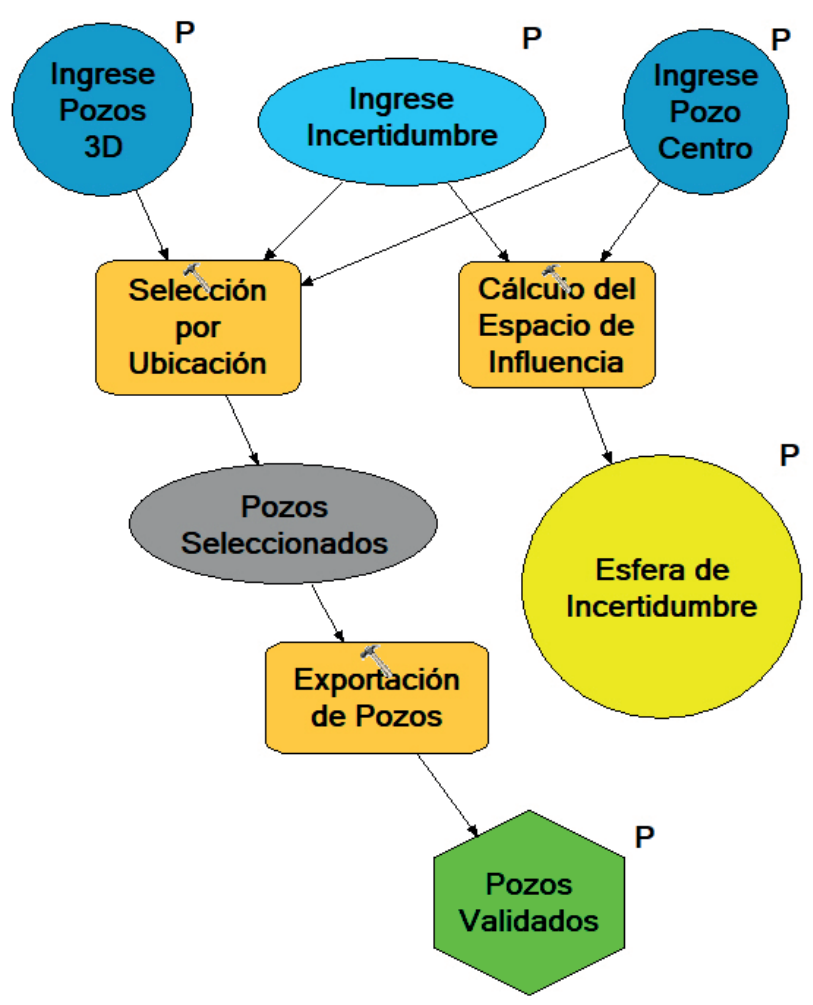

Fig. 6. Constructor de modelos de Uncertainty Sphere

\section{Caso de Estudio}

El Perú, en donde se han perforado 1996 pozos exploratorios distribuidos en 71 lotes petroleros (Perupetro, 2017), se encuentra ubicado en el hemisferio sur del planeta y se halla dividido en tres zonas geográficos (husos): 17, 18 y 19; y en tres bandas geográficas: K, L y M. La investigación se ha centrado en la cuadrícula central superior correspondiente a la zona 18 y la banda $\mathrm{M}$, donde se han perforado 215 pozos de los cuales se han considerado como población 145 pozos correspondientes a los 20 lotes existentes en la zona (ver Figura 8). La muestra se ha establecido por estratos sobre una "n" ajustada de 105 pozos. De acuerdo con el muestreo aleatorio estratificado con criterio de afijación proporcional (ver Tabla 9), se ha considerado las siguientes variables:

Nh: número total de pozos por estrato

Wh: ponderación en cada estrato

Ph: proporción de existencia de sistema geográfico en el estrato h.

Qh: proporción de no existencia de sistema geográfico en el estrato h.

Z: valor normal a un nivel de confianza dado.

E: precisión (error máximo tolerable en la estimación del parámetro).

ne: número representativo de pozos por estrato

no: muestra previa

n: muestra ajustada

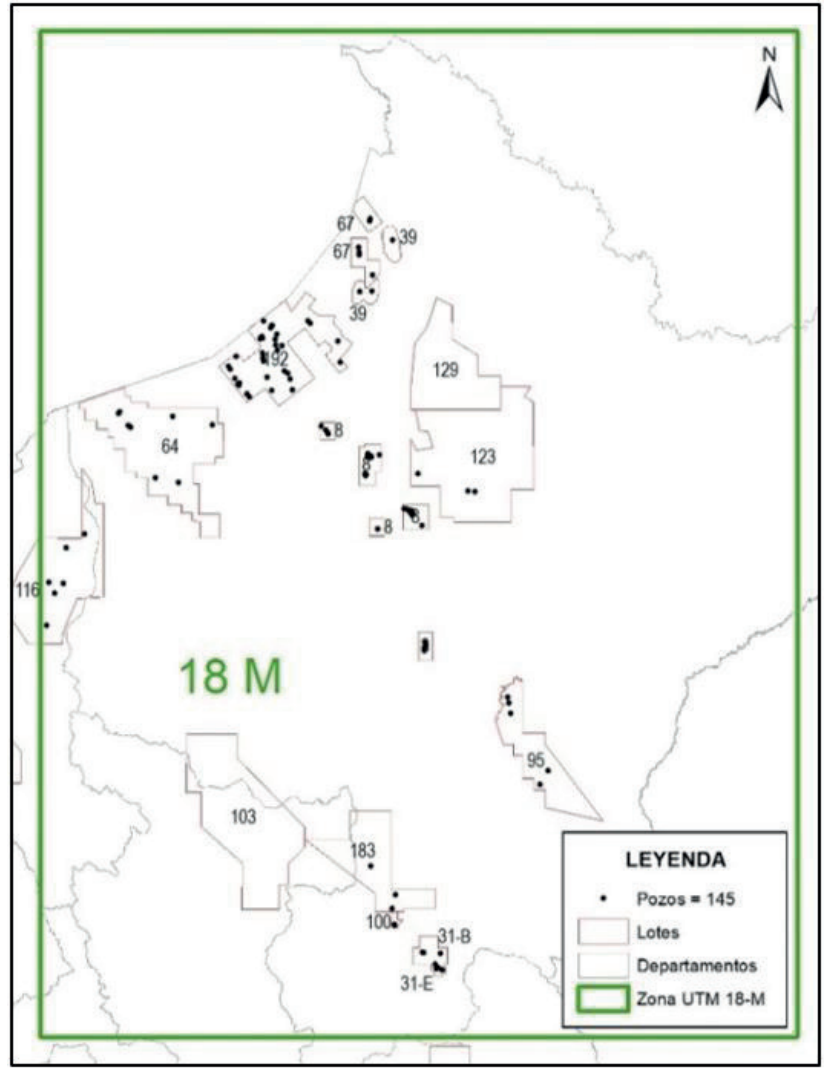

Fig. 8. Pozos ubicados en el huso 18 y zona M.

$$
\begin{gathered}
\text { no }=\frac{\left(\Sigma \mathrm{Wh}^{*} \mathrm{Ph}^{*} \mathrm{Qh}\right)}{(1+\mathrm{no} / \mathrm{N})} \\
\mathrm{n}=\frac{\mathrm{no}}{(\mathrm{E} / \mathrm{Z})^{2}}
\end{gathered}
$$

De esta manera, se han establecido como base los pozos pertenecientes al lote 192 y se han elegido en forma aleatoria 30 pozos de los 42 perforados. (ver Tabla 10). 


\subsection{Nivel Inteligencia Distribuida}

Se han tomado las coordenadas usando la aplicación GPSLogger con un celular inteligente de la marca Huawei. Además, se ha enviado los archivos generados en formato GPX en tiempo real, a través de la mensajería de WhatsApp a un segundo terminal móvil en oficina. A continuación, se muestra la información de las coordenadas, índices de confiabilidad, precisión y peso del pozo Capahuari Sur 3 usando la aplicación GPSLogger (ver Tabla 11). Paralelamente se ha obtenido las coordenadas del mismo pozo Capahuari Sur 3 haciendo uso de unidad GPS marca Garmin modelo GPSMap60CSx. Esta información se ha transmitido a la oficina vía correo electrónico. A continuación, se muestra la información de las coordenadas, los índices de confiabilidad, precisión y peso de un pozo usando el dispositivo GPSMap 60CSx (ver Tabla 12).

\subsection{Análisis y uso de la Esfera de Incertidumbre}

Con el fin de validar el método propuesto se ha revisado la información procedente de la fuente oficial de pozos de la base de datos de Perupetro y se ha procesado la información de la Inteligencia Distribuida de la siguiente manera:

Se ha recolectado " $\mathrm{n}$ " mediciones independientes y repetitivas de las coordenadas de cada uno de los pozos seleccionados del Lote 192.

$$
\mathrm{n}=20
$$

Se ha calculado los Grados de Libertad (g)

$$
\mathrm{g}=57
$$

En el cálculo del promedio $(\bar{p})$ se ha introducido la variable "Peso (W)" producto de los índices de confiabilidad anteriormente definidos.

$$
\bar{p}=\frac{1}{n W} \sum_{k}^{n}=1 q k
$$

y con la desviación estándar del Pozo Capahuari Sur 3 se ha calculado la Incertidumbre Estándar de Tipo $\mathrm{A}\left(\mu_{a}\right)$

$$
\mu_{a}=3.93
$$

Para la Incertidumbre Estándar de Tipo B, se ha calculado los siguientes grados de libertad (l)

$$
1=19 \quad(14)
$$

Con respecto a la precisión de los equipos utilizados, se han usado: Equipos GarminMap60CSx $=10 \mathrm{~m}$ y celulares de gama alta Huawei $=5 \mathrm{~m}$.

Con estos datos se ha calculado la Incertidumbre Estándar de Tipo B $(\mu b)$.

$$
\mu b=5
$$

y con las incertidumbres anteriores se ha calculado la Incertidumbre Estándar Combinada $(\mu)$.

$$
\mu_{c}=6.36
$$

además, se ha calculado el Número Efectivo de Grados de Libertad (Veff).

$$
\text { Veff }=44.09
$$

Y se ha hallado el Factor de Cobertura (K).

$$
\mathrm{K}=2.02
$$

Y finalmente se ha encontrado la Incertidumbre Expandida $(\mu e)$.

$$
\mu e=12.81 \quad(19)
$$

Con esta información se ha definido dos nuevos elementos geográficos: la "Esfera de Incertidumbre" que es el espacio geométrico que delimita la indeterminación de la posición de los elementos a partir de la ubicación encontrada en la documentación de Perupetro y, los "Pozos Válidos" que representan a dichos elementos inmersos en la Esfera de Incertidumbre generados a través de la Información Geográfica Voluntaria en su nivel de Inteligencia Distribuida usando GPSLogger y/o GarminMap60CSx (ver Figura 9).

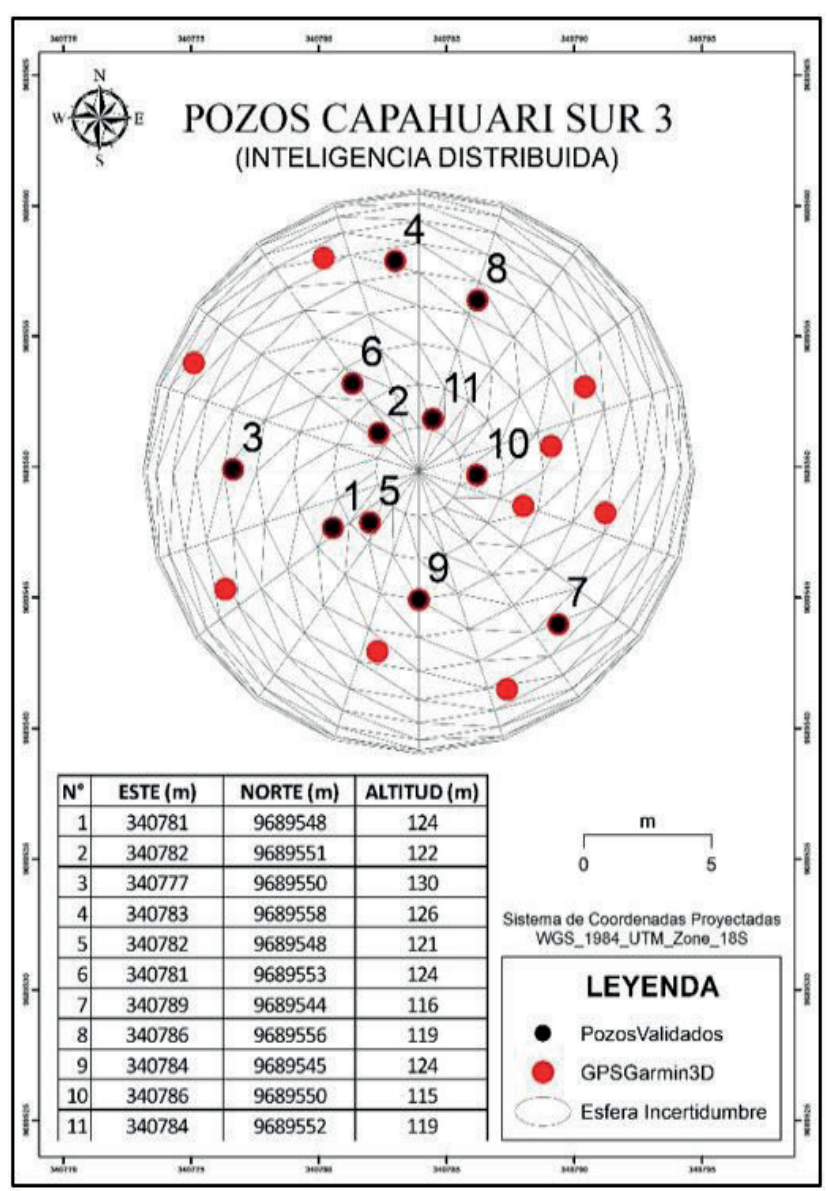

Fig. 9. Pozos Validados en la Esfera de Incertidumbre. 
Tabla 9. Muestra de pozos por estratos

\begin{tabular}{cccccccc} 
Orden & Lote & Nh & Ph & Qh & Wh=Nh/N & PhxQhxWh & ne \\
1 & 8 & 51 & 0.5 & 0.5 & 0.351724138 & 0.08793103 & 37 \\
2 & $31-B$ & 6 & 0.5 & 0.5 & 0.04137931 & 0.01034483 & 4 \\
3 & $31-\mathrm{E}$ & 5 & 0.5 & 0.5 & 0.034482759 & 0.00862069 & 4 \\
4 & 39 & 3 & 0.5 & 0.5 & 0.020689655 & 0.00517241 & 2 \\
5 & 64 & 10 & 0.5 & 0.5 & 0.068965517 & 0.01724138 & 7 \\
6 & 67 & 7 & 0.5 & 0.5 & 0.048275862 & 0.01206897 & 5 \\
7 & 95 & 5 & 0.5 & 0.5 & 0.034482759 & 0.00862069 & 4 \\
8 & 100 & 4 & 0.5 & 0.5 & 0.027586207 & 0.00689655 & 3 \\
9 & 103 & 0 & 0.5 & 0.5 & 0 & 0 & 0 \\
10 & 116 & 6 & 0.5 & 0.5 & 0.04137931 & 0.01034483 & 4 \\
11 & 123 & 3 & 0.5 & 0.5 & 0.020689655 & 0.00517241 & 2 \\
12 & 129 & 0 & 0.5 & 0.5 & 0 & 0 & 0 \\
13 & 183 & 3 & 0.5 & 0.5 & 0.020689655 & 0.00517241 & 2 \\
14 & 192 & 42 & 0.5 & 0.5 & 0.289655172 & 0.07241379 & 30 \\
N= & 145 & Z= & 1.96 & & $\mathbf{E P h} \times \mathbf{Q h} \times \mathbf{W h}=$ & 0.25 & \\
E= & 0.05 & no= & 384.16 & & $\mathrm{n}=$ & 105 & \\
\hline
\end{tabular}

Tabla 10. Muestra de pozos del lote 192.

\begin{tabular}{|c|c|c|c|c|c|c|}
\hline $\mathbf{N}^{\circ}$ & UWI & POzo & YACIMIENTO & ESTE (m) & NORTE (m) & PROF. (m) \\
\hline 1 & 1A_13 & CAPAHUARI S 3 & CAPAHUARI SUR & 340782 & 9689549 & 3911 \\
\hline 2 & 1A_26 & CAPAHUARI S 4 & CAPAHUARI SUR & 341789 & 9688457 & 4040 \\
\hline 3 & 1A_1 & CAPAHUARI N 01 & CAPAHUARI NORTE & 334077 & 9702603 & 4297 \\
\hline 4 & 1A_9 & CAPAHUARI N 02 & CAPAHUARI NORTE & 332418 & 9705180 & 5579 \\
\hline 5 & $1 \mathrm{~A} \_2$ & CAPAHUARI S 01X & CAPAHUARI SUR & 341947 & 9690353 & 4263 \\
\hline 6 & $1 \mathrm{~A} 167$ & CAPAHUARI S 31 & CAPAHUARI SUR & 341790 & 9690223 & 3901 \\
\hline 7 & 1A_17 & CARMEN 1 & CARMEN & 360544 & 9729441 & 3330 \\
\hline 8 & 1A_57 & DORISSA 1 & DORISSA & 366270 & 9695562 & 11517 \\
\hline 9 & 1A_18 & FORESTAL EXT 1 & FORESTAL & 363467 & 9745259 & 10600 \\
\hline 10 & 1A_3 & FORESTAL 1 & FORESTAL & 370933 & 9740963 & 3658 \\
\hline 11 & 1A_8 & FORESTAL 2 & FORESTAL & 369718 & 9738820 & 3133 \\
\hline 12 & 1A_27 & FORESTAL 3 & FORESTAL & 370499 & 9741196 & 3133 \\
\hline 13 & 1A_29 & FORESTAL 4 & FORESTAL & 370500 & 9741194 & 3110 \\
\hline 14 & 1A_30 & FORESTAL 5 & FORESTAL & 370497 & 9741198 & 3103 \\
\hline 15 & 1A_19 & HUAYURI N 1 & HUAYURI NORTE & 362321 & 9716792 & 11165 \\
\hline 16 & 1A_33 & HUAYURI S 1 & HUAYURI NORTE & 363560 & 9709789 & 10916 \\
\hline 17 & 1A_35 & HUAYURI S 2 & HUAYURI NORTE & 363561 & 9709787 & 11180 \\
\hline 18 & 1A_36 & HUAYURI S 3 & HUAYURI NORTE & 363054 & 9712305 & 10948 \\
\hline 19 & 1A_38 & HUAYURI S 4 & HUAYURI NORTE & 363054 & 9712303 & 11203 \\
\hline 20 & 1A_40 & HUAYURI S 5 & HUAYURI NORTE & 363055 & 9712308 & 12500 \\
\hline 21 & 1B_27 & JIBARITO 1 & JIBARITO & 386699 & 9693967 & 3244 \\
\hline 22 & 1B_1 & JIBARO 1 & JIBARO & 384703 & 9699101 & 3898 \\
\hline 23 & $1 \mathrm{~A} 166$ & JIBARO EXT $1 X$ & JIBARO & 381692 & 9701382 & 3224 \\
\hline 24 & $1 \mathrm{~A} 165$ & MACUSARI 01X & MACUSANI & 339272 & 9714013 & 12855 \\
\hline 25 & $1 \mathrm{~A} 162$ & PILAR 1X & PILAR & 379899 & 9723511 & 3140 \\
\hline 26 & 1A_31 & SHIVIYACU NE1 & SHIVIYACU & 375008 & 9733734 & 3329 \\
\hline 27 & $1 \mathrm{~A} 161$ & SHIVIYACU SE1 & SHIVIYACU & 375730 & 9719364 & 3203 \\
\hline 28 & 1A_5 & SHIVIYACU 1 & SHIVIYACU & 373879 & 9724120 & 3324 \\
\hline 29 & 1A_10 & SHIVIYACU 3 & SHIVIYACU & 373370 & 9728914 & 3137 \\
\hline 30 & 1A_4 & TAMBO 01X & TAMBO & 348851 & 9681102 & 4258 \\
\hline
\end{tabular}


Tabla 11. Coordenadas, índices, precisión, peso(GPSLogger)

\begin{tabular}{|c|c|c|c|c|c|c|c|c|}
\hline $\mathbf{N}^{\circ}$ & ESTE (m) & NORTE (m) & ALTITUD (m) & PRECISION & INDICE 1 & INDICE 2 & INDICE 3 & PESO \\
\hline 1 & 340784 & 9689542 & 135 & 5 & 4 & 2 & 4 & 0.2 \\
\hline 2 & 340794 & 9689551 & 106 & 5 & 4 & 2 & 4 & 0.2 \\
\hline 3 & 340781 & 9689557 & 125 & 5 & 4 & 2 & 4 & 0.2 \\
\hline 4 & 340782 & 9689534 & 169 & 5 & 4 & 2 & 4 & 0.2 \\
\hline 5 & 340766 & 9689557 & 185 & 5 & 4 & 2 & 4 & 0.2 \\
\hline 6 & 340776 & 9689549 & 131 & 5 & 4 & 2 & 4 & 0.2 \\
\hline 7 & 340783 & 9689554 & 122 & 5 & 4 & 2 & 4 & 0.2 \\
\hline 8 & 340793 & 9689550 & 104 & 5 & 4 & 2 & 4 & 0.2 \\
\hline 9 & 340786 & 9689556 & 119 & 5 & 4 & 2 & 4 & 0.2 \\
\hline 10 & 340793 & 9689546 & 97 & 5 & 4 & 2 & 4 & 0.2 \\
\hline 11 & 340790 & 9689560 & 130 & 5 & 4 & 2 & 4 & 0.2 \\
\hline 12 & 340778 & 9689540 & 154 & 5 & 4 & 2 & 4 & 0.2 \\
\hline 13 & 340775 & 9689563 & 187 & 5 & 4 & 2 & 4 & 0.2 \\
\hline 14 & 340787 & 9689540 & 142 & 5 & 4 & 2 & 4 & 0.2 \\
\hline 15 & 340795 & 9689540 & 110 & 5 & 4 & 2 & 4 & 0.2 \\
\hline 16 & 340780 & 9689556 & 126 & 5 & 4 & 2 & 4 & 0.2 \\
\hline 17 & 340773 & 9689533 & 174 & 5 & 4 & 2 & 4 & 0.2 \\
\hline 18 & 340775 & 9689553 & 138 & 5 & 4 & 2 & 4 & 0.2 \\
\hline 19 & 340787 & 9689546 & 115 & 5 & 4 & 2 & 4 & 0.2 \\
\hline 20 & 340796 & 9689546 & 92 & 5 & 4 & 2 & 4 & 0.2 \\
\hline
\end{tabular}

Tablas 12. Coordenadas, índices, precisión y peso (Garmin)

\begin{tabular}{|c|c|c|c|c|c|c|c|c|}
\hline $\mathbf{N}^{\circ}$ & ESTE (m) & NORTE (m) & ALTITUD (m) & PRECISION (m) & INDICE 1 & INDICE 2 & INDICE 3 & PESO \\
\hline 1 & 334077 & 9702607 & 341 & 8 & 2 & 3 & 2 & 0.13 \\
\hline 2 & 334076 & 9702606 & 336 & 9 & 2 & 3 & 2 & 0.11 \\
\hline 3 & 334076 & 9702599 & 301 & 5 & 2 & 3 & 2 & 0.20 \\
\hline 4 & 334074 & 9702597 & 262 & 8 & 2 & 3 & 2 & 0.13 \\
\hline 5 & 334070 & 9702600 & 302 & 6 & 2 & 3 & 2 & 0.17 \\
\hline 6 & 334075 & 9702599 & 294 & 7 & 2 & 3 & 2 & 0.14 \\
\hline 7 & 334085 & 9702598 & 268 & 8 & 2 & 3 & 2 & 0.13 \\
\hline 8 & 334085 & 9702600 & 286 & 5 & 2 & 3 & 2 & 0.20 \\
\hline 9 & 334079 & 9702596 & 271 & 5 & 2 & 3 & 2 & 0.20 \\
\hline 10 & 334081 & 9702599 & 292 & 5 & 2 & 3 & 2 & 0.20 \\
\hline 11 & 334081 & 9702598 & 285 & 5 & 2 & 3 & 2 & 0.20 \\
\hline 12 & 334074 & 9702596 & 253 & 7 & 2 & 3 & 2 & 0.14 \\
\hline 13 & 334077 & 9702601 & 321 & 6 & 2 & 3 & 2 & 0.17 \\
\hline 14 & 334074 & 9702612 & 324 & 6 & 2 & 3 & 2 & 0.17 \\
\hline 15 & 334074 & 9702612 & 329 & 9 & 2 & 3 & 2 & 0.11 \\
\hline 16 & 334087 & 9702603 & 306 & 5 & 2 & 3 & 2 & 0.20 \\
\hline 17 & 334078 & 9702613 & 361 & 5 & 2 & 3 & 2 & 0.20 \\
\hline 18 & 334077 & 9702606 & 338 & 7 & 2 & 3 & 2 & 0.14 \\
\hline 19 & 334075 & 9702598 & 280 & 7 & 2 & 3 & 2 & 0.14 \\
\hline 20 & 334077 & 9702603 & 333 & 8 & 2 & 3 & 2 & 0.13 \\
\hline
\end{tabular}




\section{Resultados}

A partir de las Esferas de Incertidumbre y usando el método de Información Geográfica Voluntaria en su nivel de Inteligencia Distributiva, se han validado: seis pozos con el GPSLogger (ver Tabla 13) y once pozos con el GPSMap60CSx (ver Tabla 14).

Tabla 13. Pozos validados usando GPSLogger

\begin{tabular}{cccc}
$\mathbf{N}^{\circ}$ & ESTE $(\mathbf{m})$ & NORTE $(\mathrm{m})$ & ALTITUD $(\mathrm{m})$ \\
\hline 1 & 340784 & 9689542 & 135 \\
2 & 340781 & 9689557 & 125 \\
3 & 340776 & 9689549 & 131 \\
4 & 340783 & 9689554 & 122 \\
5 & 340780 & 9689556 & 126 \\
6 & 340775 & 9689553 & 138 \\
\hline
\end{tabular}

Tabla 14. Pozos validados usando la Inteligencia Distribuida

\begin{tabular}{cccc}
$\mathbf{N}^{\circ}$ & ESTE $(\mathrm{m})$ & NORTE $(\mathrm{m})$ & ALTITUD $(\mathrm{m})$ \\
\hline 1 & 340781 & 9689548 & 124 \\
2 & 340782 & 9689551 & 122 \\
3 & 340777 & 9689550 & 130 \\
4 & 340783 & 9689558 & 126 \\
5 & 340782 & 9689548 & 121 \\
6 & 340781 & 9689553 & 124 \\
7 & 340789 & 9689544 & 116 \\
8 & 340786 & 9689556 & 119 \\
9 & 340784 & 9689545 & 124 \\
10 & 340786 & 9689550 & 115 \\
11 & 340784 & 9689552 & 119 \\
\hline
\end{tabular}

Se ha considerado ahora la ubicación dada por la documentación de Perupetro y las ubicaciones de los puntos centrales medios ponderados de las Esferas de Incertidumbre, obtenidas a través de la Inteligencia Distributiva con GPSLogger y GPSGarmin, para determinar que los desfases de ubicación con respecto a las coordenadas oficiales son más que aceptables, en función de la mejor estimación del valor atribuible calculada con la Incertidumbre Expandida (IE) para las coordenadas de la Inteligencia Distributiva (ver Tabla 15).

Tabla 15. Mejor estimación de las coordenadas de pozo.

\begin{tabular}{lll} 
POZO & $\begin{array}{l}\text { GPSLOGGER } \\
+/ \text {-IE }\end{array}$ & $\begin{array}{l}\text { GPSGARMIN } \\
+/ \text {-IE }\end{array}$ \\
& $\mathrm{X}=340783.69$ & $\mathrm{X}=340783.90$ \\
& $+/-12.81$ & +-10.79 \\
CAPAHUARI & & $\mathrm{Y}=9689549.83$ \\
SUR 3 & $\mathrm{Y}=9689548.70$ & $+/-10.79$ \\
& $+/-12.81$ & \\
\hline
\end{tabular}

\section{Conclusiones}

A través del estado del arte, donde se ha revisado diversos métodos para mejorar el geoposicionamiento, se ha concluido que el Método de Geodatabase es el adecuado tanto para el geoprocesamiento de la incertidumbre como para el almacenamiento de la información validada.

El estudio de los distintos métodos de procesamiento de datos y de la Guía para la expresión de la Incertidumbre de la medida (GUM), ha permitido concluir que la mejor manera de determinar los Índices de confiabilidad para las fuentes de incertidumbres analizadas en este trabajo, están en función de la precisión del dispositivo, equipamiento o procedimiento con que se han realizado las mediciones.

Además, se ha concluido que el uso de los smartphones con el aplicativo propuesto GPSLogger, aporta valiosa información que complementa a la información oficial del espacio geográfico en estudio. Si bien la información Geográfica Voluntaria es hoy en día, una fuente valiosa para los distintos tipos de investigaciones, es necesario definir el nivel, o niveles, sobre el cual recolectar los datos. En la presente investigación, el trabajo en el primer nivel (crowdsourcing) con los pobladores de la zona ha sido imposible debido a la falta de equipos (smartphones), a la limitada cobertura de internet, al desinterés de los pobladores y a la falta de acceso a las instalaciones petroleras. Por lo tanto, se ha optado por trabajar sobre el segundo nivel (Inteligencia Distribuida) con personal no relacionado directamente con la empresa (proveedores) y con personal de la empresa del área de Responsabilidad Social que, si contaban con equipos y con cobertura de internet, obteniendo de esta manera una nueva fuente de información.

Con respecto al análisis de la incertidumbre, se ha utilizado el cálculo de la Incertidumbre Expandida para definir a la "Esfera de Incertidumbre", que es el entorno que alberga la dispersión de las posibles ubicaciones que podrían ser razonablemente atribuidos a la posición real del pozo exploratorio petrolero. Además, el desarrollo del artefacto "Uncertainty Sphere" creado con Python 2.7 dentro del programa geográfico ArcGIS 10.5, ha permitido que sea compatible con otros softwares de código libre.

\section{Trabajo Futuro}

Dentro de la misma línea de investigación, los estudios geológicos, geoquímicos, atmosféricos y microbiólogos del área a perforar, deberán considerar su incertidumbre asociada y, por ende, la generación de nuevos índices de confiablidad.

El mayor avance del crowdsourcing y, en particular, la información geográfica voluntaria, requerirá también un desarrollo más formal para determinar niveles estándar de confianza que puedan usarse a nivel global. 
La creación de una guía y posteriormente de un ISO, serían los siguientes pasos.

Si bien el análisis de la incertidumbre muestra en forma general el mesurando y su dispersión tridimensional, una revisión a diferentes niveles de confianza todavía puede ser profundizado.

Finalmente, la mayor precisión de los sistemas de navegación registradas en los chips de los celulares, aumentará la precisión a nivel de $1 \mathrm{~m}$ o hasta submétricos, por su compatibilidad no solo con el GPS americano y el Galileo europeo, sino también con el GLONASS ruso, con el Beidou chino y con el recién estrenado QZSS japonés.

\section{Expresiones de gratitud}

Se agradece a la plana docente de la Maestría de Sistemas - GTIC de la Facultad de Ingeniería de Sistemas e Informática de la Universidad Nacional Mayor de San Marcos y en forma particular, al Dr. Glen Rodríguez Rafael y al Mg. Joel Mercado Rojas, quienes marcaron las directrices necesarias para llevar a cabo este trabajo. Se agradece también al grupo de investigación: "The Expendables" por su valiosa ayuda para mejorar este manuscrito. Asimismo se agradece a la empresa Frontera Energy por facilitar el acceso a su base de datos petrolera exploratoria y apoyar con sus equipos, proveedores y personal.

\section{Referencias}

[1] Bolaños, R. (2017). Reseña Histórica de la Exploración por Petróleo en las Cuencas Costeras del Perú. Boletín de la Sociedad Geológica del Perú, v. 112, p. 001-013

[2] Perez-Taiman, J. (2009). Breve reseña de la exploración y explotación de petróleo en el Perú desde el punto de vista legal. Círculo de derecho administrativo. Recuperado de http://revistas.pucp.edu.pe/index.php/derechoadministrativo/ article/view/13994

[3] [3] Delgado, Tatiana \& Crompvoets, Joep. (2007). Proyecto CYTED - IDEDES 606PI0294 "Evaluación y Potenciación de Infraestructuras de Datos Espaciales para el desarrollo sostenible en América Latina y el Caribe", Infraestructuras de Datos Espaciales en Iberoamérica y el Caribe. (Ed): IDICT, Habana, Cuba, 2007), pp. 14-24

[4] GEOIDEP. (2017). Infraestructura de Datos Espaciales del Perú. Avances y estrategia de implementación al 2021. Recuperado 31 abril, 2018, de http://www.geoidep.gob.pe/ mapeandoperu/presentaciones2017/01-01-SEGDI-Avances-estrategia-perspectivas-IDEP-2021.pdf

[5] Bickel, J. y Bratvold, R. (2007). Decision Making in the Oil and Gas Industry: From Blissful Ignorance to Uncertainty-Induced Confusion. Paper SPE 109610 presented at the SPE Annual Technical Conference and Exhibition, Anaheim, California, 11-14 November. http://dx.doi.org/10.2118/109610-MS

[6] BickMcVay, D. y Dossary, M. (2014). The Value of Assessing Uncertainty. Society of Petroleum Engineers. doi: 10.2118/160189-PA

[7] Suslick, S., Schiozer, D. y Rodriguez, M. (2009). Uncertainty and risk analysis in petroleum exploration and production.
Terræ. (6), 30 - 41. Recuperado de https://www.researchgate. net/publication/285137122_Uncertainty_and_risk_analysis_ in_petroleum_exploration_and_production

[8] ESRI (Environmental Systems Research Institute). The Power of Mapping.What is GIS? Recuperado de http://www.esri. com/what-is-gis

[9] Burrough, P.A. (1986) Principles of Geographic Information Systems for Land Resource Assessment. Monographs on Soil and Resources Survey No. 12, Oxford Science Publications, New York.

[10] NCGIA, National Center for Geographic Information and Analysis (1989) Cowen, D. (1989). What is a GIS? Recuperado de http://www.geog.ubc.ca/courses/klink/gis. notes/ncgia/ u01.html\#SEC1.1.2

[11] Goodchild, M. F. (2007). Citizens as sensors: Web 2.0 and the volunteering of geographic information. GeoFocus, 7, 8-10.

[12] Haklay, M., (2013). Citizen Science and Volunteered Geographic Information - overview and typology of participation in Sui, D.Z., Elwood, S. and M.F. Goodchild (eds.), 2013. Crowdsourcing Geographic Knowledge: Volunteered Geographic Information (VGI) in Theory and Practice. Berlin: Springer. 105-122 doi: 10.1007/978-94-007-4587-2_7

[13] Evaluation of measurement data - Guide to the expression of Uncertainty in Measurement (GUM). Edición digital. https://www.bipm.org/utils/common/documents/jcgm/JCGM_100_2008_E.pdf

[14] AENOR (2014). UNE-EN ISO 19157:2014 Información Geográfica - Calidad de datos. AENOR.

[15] Blackmond L., K., Wright, E., Da Costa, P. (2010), Envisioning uncertainty in geospatial information. International Journal of Approximate Reasoning, 51, 209-223, doi.org/10.1016/j. ijar.2009.05.011

[16] GoodBan, H. y Ahlqvist, O. (2009). Representing and negotiating uncertain geospatial concepts Where are the exurban areas?, Computers, Environment and Urban Systems, 33, 233-246, doi.org/10.1016/j.compenvurbsys. 2008.10.001

[17] Viard, T., Caumon, G. y Lévy, B. (2011). Adjacent versus coincident representations of geospatial uncertainty: Which promote better decisions?, Computers\&Geosciences, 37, 511-520, doi.org/10.1016/j.cageo.2010.08.004

[18] Tavana, M., Liu, W., Elmore, P., Petry, F. et al. (2016). A practical taxonomy of methods and literature for managing uncertain spatial data in geographic information systems. Measurement, 81, 123-162, doi.org/ 10.1016/..measurement.2015.12.007

[19] Soler, T., Han, J.Y. y Weston N.D. (2011). Alternative transformation from Cartesian to geodetic coordinates by least squares for GPS georeferencing applications. Computers \& Geosciences, 42, 100-109, doi:10.1016/j.cageo.2011.10.026

[20] Zhao, H., Zhang B., Wuc, C et al. (2013). Development of a Coordinate Transformation method for direct georeferencing in map projection frames. ISPRS Journal of Photogrammetry and Remote Sensing, 77, 94-103, doi: 10.1016/j.isprsjprs.2012.12.004

[21] Civicioglu P. (2011). Transforming geocentric cartesian coordinates to geodetic coordinates by using differential search algorithm. Computers \& Geosciences, 46, 229-247, doi:10.1016/j.cageo.2011.12.011 
[22] Wu, C. y Su, W. (2012). Lattice-based clustering and genetic programming for coordinate transformation in GPS applications. Computers \& Geosciences, 52, 85-94, doi:10.1016/j. cageo.2012.09.022

[23] Breunig M., Kuper P., Butwilowski E. et al. (2015). The story of DB4GeO - A service-based geo-database architecture to support multi-dimensional data analysis and isualization. ISPRS Journal of Photogrammetry and Remote Sensing, 117, 187-205, doi:10.1016/ j.isprsjprs. 2015.12.006

[24] Kingdon A., Nayembil M. L., Richardson A. E. et al. (2016). A geodata warehouse: Using denormalisation techniques as a tool for delivering spatially enabled integrated geological information to geologists. Computers \& Geosciences, 96, 87 97, doi:10.1016/j.cageo.2016.07.016

[25] Chesnaux, R., Lambert, M., Walter, J. et al. (2011). Building a geodatabase for mapping hydrogeological features and 3D modeling of groundwater systems: Application to the Saguenay-Lac-St.-Jean region, Canada. Computers \& Geosciences, 37 (11), $1870-1882$, doi:10.1016/j.cageo.2011.04.013

[26] Hu Y., Chen H., Li G. et al. (2015). A statistical training data cleaning strategy for the PCA-based chillersensor fault detection, diagnosis and data reconstruction method. Energy and Buildings, 112, 270-278, doi:10.1016/j.enbuild.2015.11.066

[27] Saberi B. y Ghadiri N. (2016). A Sample-Based Approach to Data Quality Assessment in Spatial Databases with Application to Mobile Trajectory Nearest-Neighbor Search. Journal of Computing and Security, arXiv:1409.2819
[28] Baura, J., Moreno-Villanueva, M., Kötterb, T., Sindlingera, T. et al. (2015). MARK-AGE data management: Cleaning, exploration and visualization of data. Mechanisms of Ageing and Development, 151, 38-44, doi:10.1016/j. mad.2015.05.007

[29] Heipke, C. (2010). Crowdsourcing geospatial data. ISPRS Journal of Photogrammetry and Remote Sensing, 65, 550557, doi:10.1016/j.isprsjprs.2010.06.005

[30] Thiele, S., Jessell, M., Lindsay, M. et al. (2016). The topology of geology 1: Topological analysis, Journal of Structural Geology, 91, 27-38, doi.org /10.1016/ j.jsg. 2016.08.009

[31] Rezaei, J. (2015). Best-worst multi-criteria decision-making method. Omega, 53, $49-57$, doi.org/ 10.1016 /j. omega.2014.11.009

[32] Zeiler, M. Modeling Our World. The ESRI Guide to Geodatabase Design. Redlands. 1999. ESRI Press. 199 p.

[33] Ta-Kang Yeh (2015). Calibration of the GNSS Receivers - Methods, Results and Evaluation, Satellite Positioning Shuanggen Jin, IntechOpen, doi: 10.5772/ 58887. Recuperado de https://www.intechopen.com /books/ satellite-positioning-methods-models-and-applications/calib ration-of-the-gnss-receivers-methods-results-and-evaluation

[34] Python. What is Python? Documentation. Executive Summary. Recuperado de https://www.python.org/doc/ essays/ blurb/ 